\title{
The efficacy of laser-assisted in-office bleaching and home bleaching on sound and demineralized enamel
}

\author{
Farzaneh Ahrari ${ }^{1}$, Majid Akbari ${ }^{2}$, Sakineh Mohammadpour ${ }^{3}$, Maryam Forghani ${ }^{4}$ \\ 1: Dental Research Center, Department of Orthodontics, School of Dentistry, \\ Mashbad University of Medical Sciences, Mashbad, Iran \\ 2: Dental Research Center, Department of Operative dentistry, School of Dentistry, \\ Mashbad University of Medical Sciences, Mashbad, Iran \\ 3: Postgraduate Dental Student, Department of Periodontics, School of Dentistry, \\ Babol University of Medical Sciences, Babol, Iran \\ 4: Dental Materials Research Center, Department of Endodontics, School of Dentistry, \\ Mashbad University of Medical Sciences, Mashbad, Iran
}

\begin{abstract}
Aims: This study investigated the effectiveness of laser-assisted in-office bleaching and homebleaching in sound and demineralized enamel.

Materials and Methods: The sample consisted of 120 freshly-extracted bovine incisors. Half of the specimens were stored in a demineralizing solution to induce white spot lesions. Following exposure to a tea solution for 7.5 days, the specimens were randomly assigned to 4 groups of 30 according to the type of enamel and the bleaching procedure employed. Groups 1 and 2 consisted of demineralized teeth subjected to in-office bleaching and home bleaching, whereas in groups 3 and 4 , sound teeth were subjected to in-office and home bleaching, respectively. A diode laser (810 nm, $2 \mathrm{~W}$, continuous wave, four times for 15 seconds each) was employed for assisting the in-office process. The color of the specimens was measured before (T1) and after (T2) staining and during (T3) and after (T4) the bleaching procedures using a spectrophotometer. The color change $(\Delta \mathrm{E})$ between different treatments stages was compared among the groups.

Results: There were significant differences in the color change between T2 and T3 ( $\Delta \mathrm{E}$ T2-T3) and T2 and T4 $(\Delta \mathrm{E}$ T2-T4) stages among the study groups $(\mathrm{p}<0.05)$. Pairwise comparison by Duncan test revealed that both $\Delta$ ET2-T3 and $\Delta$ ET2-T4 were significantly greater in demineralized teeth submitted to laser-assisted in-office bleaching (group 1) as compared to the other groups $(\mathrm{P}<0.05$ ).

Conclusion: Laser-assisted in-office bleaching could provide faster and greater whitening effect than home bleaching on stained demineralized enamel, but both procedures produced comparable results on sound teeth.
\end{abstract}

Key words: In office bleaching $\cdot$ home bleaching $\cdot$ demineralized enamel $\cdot$ white spot lesion • laser-spectrophotometry $\cdot$ Diode $\cdot$ Tooth color

\section{Introduction}

Demineralization areas called "white spots" are frequently observed in subjects with high risk of caries

Addressee for Correspondence:

First Corresponding Author:

Maryam Forghani

Dental Materials Research Center, School of Dentistry,

Mashhad University of Medical Sciences, Vakilabad

Boulevard, Mashhad, Iran

Phone: +98-513-8829501 Fax: 05138829500

E-mail: forghaniradm@mums.ac.ir

forghanirad_m@yahoo.com

Second Corresponding Author:

Farzaneh Ahrari

E-mail: Ahrarif@mums.ac.ir such as those undergoing fixed orthodontic therapy. 1) These spots are the first clinical signs of demineralization and are characterized by their opacity compared to healthy enamel. ${ }^{2)}$ The milky color of white spots may impair the appearance of the teeth and make the patients unsatisfied with the results of esthetic dental treatments. ${ }^{3)}$ Different approaches have been used for managing white spot lesions including the application of products containing fluoride or casein phosphopeptide-amorphous calcium phosphate (CPP-ACP), microabrasion, bleaching and restorative treatments. 4,5) Bleaching has been employed as a minimally inva-

Received date: April 9h, 2015

Accepted date: August 18th, 2015 
sive technique to mask the lesions by achieving a more uniform appearance throughout the dentition. It has been demonstrated that white spots match better with the remaining tooth structure following the bleaching procedures. ${ }^{5-8)}$ Knosel et al. ${ }^{8}$ ) found that bleaching could satisfactorily camouflage white spot lesions observable following fixed orthodontic therapy. The bleaching treatment may have additional advantages in orthodontically treated subjects as they are usually affected with various degrees of discoloration in healthy and demineralized parts of the dentition.

There are two main methods of professional dental bleaching; home applied and in-office procedures. Home bleaching involves the application of bleaching agents that liberate low levels of hydrogen peroxide (HP) over a long period of time. In contrast, in-office bleaching employs much higher levels of HP (up to $55 \%$ ) for short durations. 9) The use of home-bleaching is associated with several advantages such as less HP concentration, lower cost and taking less time in the dental office, ${ }^{10,11)}$ but in this procedure, there is lack of control on the use of bleaching agents and trays by the patients. The main advantages of in-office bleaching include dental practitioner control on the bleaching process, prevention of soft-tissue exposure or material ingestion, reduced treatment time and immediate results that enhance patient's satisfaction with dental treatment. ${ }^{12)}$

The in-office procedures could be accelerated by the application of light or heat to activate the bleaching agent. Several types of activation devices are available to be used in combination with bleaching materials such as halogen curing lamps, plasma arc lamps, lightemitting diodes (LEDs) and lasers. Laser radiation assists the release of free radicals in the bleaching agents, leading to a faster whitening process. Furthermore, it has been demonstrated that laser irradiation could minimize hypersensitivity of the teeth during the bleaching process. ${ }^{13}$, 14) One important concern about using the peroxide-based formulations is alteration in surface micromorphology and reduction in microhardness and calcium content of enamel. ${ }^{14,15)}$ It has been claimed that diode laser activation of HP during the bleaching process not only increases the whitening effect but also protects the change in enamel structure compared to that occurs with gel treatment alone. ${ }^{16)}$

There are a few studies regarding comparison of the two professional bleaching techniques on managing discolored white spot lesions. The present study aimed to evaluate, through the spectrophotometric analysis, the effectiveness of laser-assisted in-office bleaching and home-bleaching in sound and demineralized enamel.

\section{Materials and Methods}

The sample consisted of 120 freshly-extracted bovine incisors without visible caries or structural defects on the enamel surface. The teeth were immersed in a $0.1 \%$ thymol solution for 1 week at room temperature, followed by storage in distilled water until the time of the experiment. The teeth were cleaned with pumice slurry and brush. Then, half of the specimens were individually immersed in $5 \mathrm{ml}$ of a demineralizing solution for 12 weeks to create artificial caries. The demineralizing solution consisted of $2.2 \mathrm{mM} \mathrm{CaCl} 2,2.2 \mathrm{mM}$ $\mathrm{NaH} 2 \mathrm{Po} 4$ and $50 \mathrm{mM}$ acetic acid, with $\mathrm{pH}$ adjusted at 4.8 using potassium hydroxide $\left.(\mathrm{KOH}) .{ }^{17}\right)$ This solution was refreshed weekly. The sample was then randomly divided into 4 groups of 30 according to the type of enamel and the bleaching procedure employed as follows.

Group 1 consisted of demineralized teeth subjected to in-office procedure.

Group 2 consisted of demineralized teeth underwent home bleaching.

Group 3 consisted of sound teeth subjected to in-office procedure.

Group 4 consisted of sound teeth underwent home bleaching.

\section{Color change measurement}

Before bleaching, all the specimens were artificially stained using a tea solution. The tea solution was prepared by boiling $2 \mathrm{~g}$ of tea in $100 \mathrm{ml}$ of distilled water for 5 min followed by filtering through gauze to remove the tea from the infusion. ${ }^{18)}$ Each tooth was immersed in $10 \mathrm{ml}$ of this solution for 7.5 days and then rinsed thoroughly under tap water.

A spectrophotometer (Easyshade; Vita Zahnfabrik, Bad Säckingen, Germany) was used to measure the color of the specimens according to the CIELAB (Commission International de l'Eclairage $\mathrm{L}^{*} \mathrm{a}^{*}$ and $\mathrm{b}^{*}$ ) color space system. In this system, the $\mathrm{L}$ axis indicates the value or degree of lightness (ranges from 0: black to 100: white), whereas the a plane represents the degree of $\mathrm{red} /$ green $(+\mathrm{a}$ : red, $-\mathrm{a}$ : green) and the $\mathrm{b}$ plane corresponds with the degree of yellow/blue (+ b: yellow, - b: blue) within the sample. The color of the teeth was assessed before (T1, baseline examination) and after (T2) the staining procedure. The middle 
part of the tooth was considered for color measurement.

\section{The Laser-assisted in-office bleaching process}

The in-office bleaching consisted of the application of a 40\% hydrogen peroxide gel (Opalescence Xtra Boost; Ultradent Products Inc., South Jordan, UT, USA) followed by laser irradiation for further activation of the bleaching agent. The gel was prepared according to the manufacturer's instructions and was applied at a thickness of 1-2 mm on the buccal enamel surface. The apparatus employed was a gallium-aluminum-arsenide (GaAlAs) diode laser with a wavelength of $810 \mathrm{~nm}$ (ARC Laser GmbH, Nuremberg, Germany). The laser was held manually at an approximate distance of 1 $\mathrm{mm}$ from the gel and perpendicular to the enamel surface, and the irradiation was performed at power of 2 $\mathrm{W}$ and continuous wave (CW) mode, using a non-contact bleaching handpiece.

The in-office bleaching was performed for 3 sessions every seven days, in 15 days. In every session, the bleaching gel remained in contact with the specimen for a total period of 10 minutes, and the laser was irradiated four times for 15 seconds each at intervals of 2 minutes between irradiations. The total irradiation time was 60 seconds per tooth and the first laser irradiation was performed after 1 minute of placing gel on the enamel surface. After the bleaching process, the teeth were thoroughly rinsed under tap water and stored in Fuzayama Meyer artificial saliva solution. This solution consisted of $\mathrm{KCl}(0.4 \mathrm{~g} / 1), \mathrm{NaCl}(0.4 \mathrm{~g} / 1)$, $\mathrm{CaCl}_{2} .2 \mathrm{H}_{2} \mathrm{O}(0.906 \mathrm{~g} / \mathrm{l}), \mathrm{NaH}_{2} \mathrm{PO}_{4} .2 \mathrm{H}_{2} \mathrm{O}(0.690 \mathrm{~g} / \mathrm{l})$, $\mathrm{Na}_{2} \mathrm{~S} .9 \mathrm{H}_{2} \mathrm{O}(0.005 \mathrm{~g} / \mathrm{l})$ and urea $(1 \mathrm{~g} / \mathrm{l})$ with $\mathrm{pH}=7.03$.

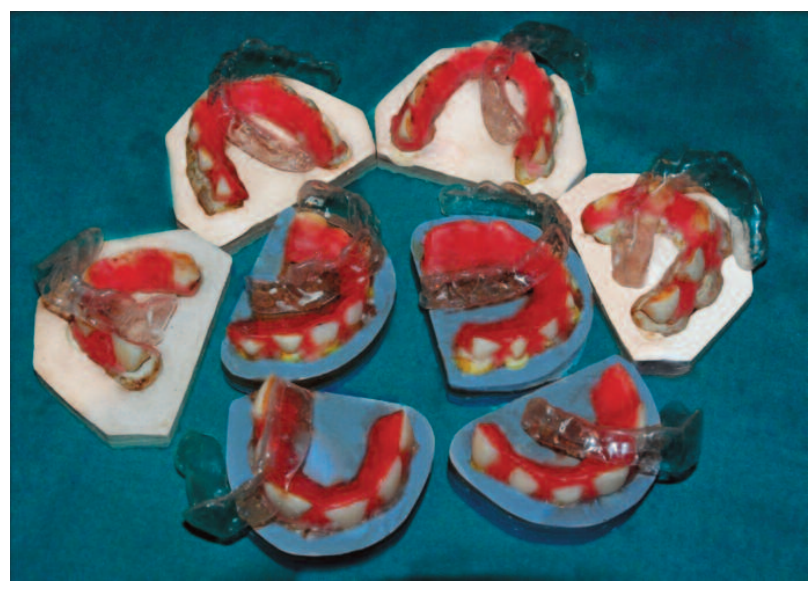

Fig. 1: Mounting the teeth in plaster for making custom trays. The undercuts have been filled out by wax.
The artificial saliva was replaced daily. The specimens were subjected to color assessment on day 1 following the second (T3) and third (T4) in-office bleaching sessions.

\section{The home bleaching procedure}

To make custom trays for the home bleaching procedure, every 6-8 teeth were mounted in plaster so that an ovoid arch was formed, resembling upper dentition (Fig 1). The undercuts were filled out by pink wax (Fig 1). The arch was then covered by a layer of pink wax to allow making a special tray. An alginate impression was then taken and a bleaching tray was made for each dentition.

A 15\% carbamide peroxide gel (Opalescence 15\%; Ultradent Products Inc.,South Jordan, UT, USA) was used for home bleaching. The gel was applied on the enamel surface of each specimen at a thickness of 1-2 $\mathrm{mm}$, and then the custom-made tray was placed over the arch. The plaster models were kept at $37^{\circ} \mathrm{C}$ and $100 \%$ humidity. After 8 hours, the gel was thoroughly rinsed off the enamel surface, and the specimens were kept in refreshed artificial saliva for the remaining hours of the day. The whitening process was repeated daily over a period of 15 days.

The specimens in the home bleaching groups were subjected to color assessments at days 8 (T3) and 16 (T4) after starting the process.

All measurements were performed twice by the same operator and the mean value was calculated. The total color change $(\Delta \mathrm{E})$ between different treatments stages was determined using the following formula: $\Delta \mathrm{E}=\left[(\Delta \mathrm{L})^{2}+(\Delta \mathrm{a})^{2}+(\Delta \mathrm{b})^{2}\right]^{0.5}$

\section{Statistical analysis}

The Kolmogrov-Smirnov test confirmed the normal distribution of the data. One way analysis of variance (ANOVA) was conducted to compare the color change $(\Delta \mathrm{E})$ obtained from the two measurements at T1 to T4 time points among the experimental groups, followed by Duncan post hoc test for pairwise comparisons. The data were analyzed by SPSS (Statistical Package for the Social Sciences, version 16.0, SPSS Inc, IL, USA) software and the significance level was predetermined at $\mathrm{p}<0.05$.

\section{Results}

Figures 2 to 4 present changes in $L^{*}$, $a^{*}$ and $b^{*}$ values in the study groups over the course of the experiment. Following staining (T2), the lightness value dropped (shifted to the dark domain) and then increased during 


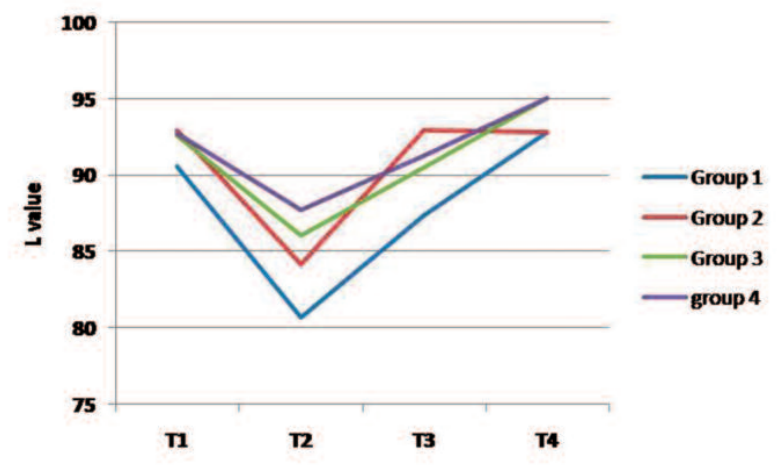

Fig. 2: A line chart indicating changes in "L" parameter in the study groups over the experiment. (Group 1 demineralized teeth subjected to inoffice bleaching, Group 2 demineralized teeth exposed to home bleaching, Group 3 sound teeth subjected to in-office procedure, Group 4 sound teeth exposed to home bleaching)

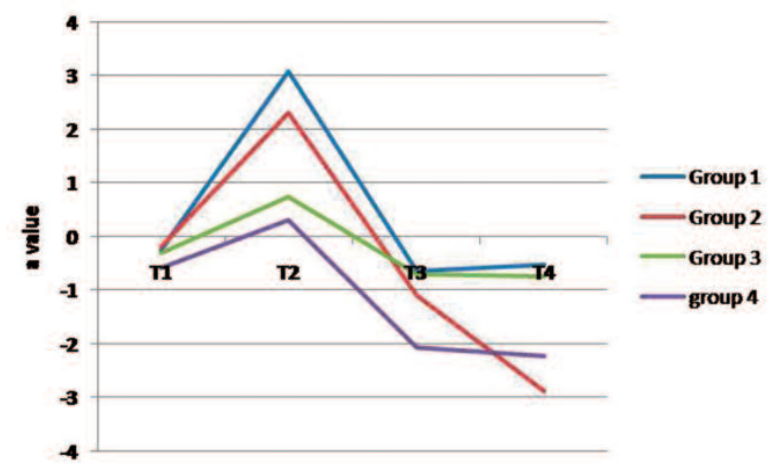

Fig. 3: A line chart indicating changes in "a" parameter in the study groups over the experiment. (Group 1 demineralized teeth subjected to inoffice bleaching, Group 2 demineralized teeth exposed to home bleaching, Group 3 sound teeth subjected to in-office procedure, Group 4 sound teeth exposed to home bleaching)

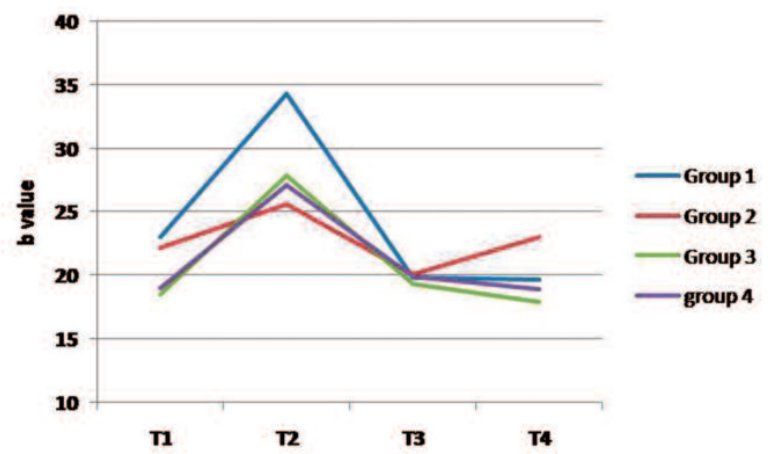

Fig. 4: A line chart indicating changes in "b" parameter in the study groups over the experiment. (Group 1 demineralized teeth subjected to inoffice bleaching, Group 2 demineralized teeth exposed to home bleaching, Group 3 sound teeth subjected to in-office procedure, Group 4 sound teeth exposed to home bleaching)
T3 and T4 stages (Fig 2). The L value of all groups (except group 2) was greater at the end of the treatment (T4) than that of the baseline examination (T1). The $\mathrm{a}^{*}$ and $\mathrm{b}^{*}$ values increased after staining (color shift to red and yellow directions, respectively) and then decreased during the bleaching therapy in most groups (Figs 3 and 4).

Table 1 indicates the descriptive statistics and the results of ANOVA and Duncan test regarding the color change $(\Delta \mathrm{E})$ between different stages in the experimental groups. No significant differences were found in the color change between T1 and T2 ( $\Delta \mathrm{ET} 1-\mathrm{T} 2), \mathrm{T} 1$ and T3 $(\Delta \mathrm{ET} 1-\mathrm{T} 3), \mathrm{T} 1$ and $\mathrm{T} 4(\Delta \mathrm{ET} 1-\mathrm{T} 4)$ and $\mathrm{T} 3$ and T4 ( $\Delta$ ET3-T4) time points ( $>>0.05$; Table 2 ) among the study groups. The experimental groups, however exhibited statistically significant differences in the color change between T2 and T3 $(\Delta \mathrm{ET} 2-\mathrm{T} 3)$ and T2 and T4 $(\Delta$ ET2-T4) intervals $(\mathrm{p}<0.05$; Table 2$)$. Pairwise comparison by Duncan test (Table 1) revealed that both $\triangle \mathrm{ET} 2-\mathrm{T} 3$ and $\Delta \mathrm{ET} 2-\mathrm{T} 4$ were significantly greater in demineralized teeth submitted to laser-assisted in-office bleaching (group 1) as compared to the other experimental groups $(\mathrm{P}<0.05)$.

\section{Discussion}

Tooth bleaching has been considered as a part of minimally invasive treatments for white spot lesions 5,7 ) which could mask the lesions and lead to a more perfect appearance and esthetic smile. In this study, a GaAlAs diode laser was used for in-office bleaching and its effect on sound and demineralized bovine teeth was compared with that of home bleaching, using color measurements. Bovine teeth are the most widely used substitute for human teeth in dental research 23) because of their close chemical composition and micro-morphological characteristics to human enamel and dentin 24, 25). Since the presence of white spot lesions is usually associated with enamel discoloration at the end of the orthodontic treatment, a staining procedure was employed in this experiment. It is believed that color change values exceeding 3.3 units are clinically visible in any site by independent observers. ${ }^{19)}$ In the present study, the color change after staining ( $\triangle \mathrm{ET} 1-\mathrm{T} 2)$ was greater than 3.3 units in the study groups, indicating that the staining stage produced observable discoloration in all the specimens.

In the present study, the $\mathrm{L}$ value decreased and the $\mathrm{a}$ and $\mathrm{b}$ values increased in all groups after immersion of the specimens in tea solution (T2). The degree of reduction in L parameter and the degree of increase in a parameter were greater in demineralized than 
Table 1: Mean and standard deviation (SD) and the results of statistical analysis for comparison of color differences $(\Delta \mathrm{E})$ between different treatment stages among the study groups (Group 1 demineralized teeth subjected to in-office bleaching, Group 2 demineralized teeth exposed to home bleaching, Group 3 sound teeth subjected to in-office procedure, Group 4 sound teeth exposed to home bleaching)

\begin{tabular}{|c|c|c|c|c|c|c|c|c|c|}
\hline \multirow{2}{*}{ Groups } & \multirow{2}{*}{ CIE } & \multicolumn{2}{|c|}{$\mathrm{T} 1$} & \multicolumn{2}{|c|}{$\mathrm{T} 2$} & \multicolumn{2}{|c|}{ T3 } & \multicolumn{2}{|c|}{$\mathrm{T} 4$} \\
\hline & & Mean & SD & Mean & SD & Mean & SD & Mean & SD \\
\hline \multirow{3}{*}{1} & $\mathrm{~L}$ & 90.55 & 3.38 & 80.68 & 4.33 & 87.41 & 17.30 & 92.83 & 3.07 \\
\hline & $\mathrm{a}$ & -0.24 & 0.86 & 3.09 & 2.07 & -0.64 & 0.67 & -0.52 & 1.26 \\
\hline & $\mathrm{b}$ & 23.03 & 4.11 & 34.28 & 3.44 & 19.81 & 3.35 & 19.66 & 3.20 \\
\hline \multirow{3}{*}{2} & $\mathrm{~L}$ & 92.95 & 3.54 & 84.19 & 12.62 & 92.95 & 3.35 & 91.80 & 12.91 \\
\hline & a & -0.20 & 1.05 & 2.30 & 2.0 & -1.11 & 1.15 & -3.88 & 11.31 \\
\hline & $\mathrm{b}$ & 22.20 & 3.55 & 25.64 & 6.04 & 20.12 & 4.12 & 23.01 & 4.19 \\
\hline \multirow{3}{*}{3} & $\mathrm{~L}$ & 92.63 & 2.83 & 86.08 & 8.14 & 90.50 & 16.37 & 95.05 & 4.30 \\
\hline & $\mathrm{a}$ & -0.30 & 1.05 & 0.75 & 2.41 & -0.70 & 1.66 & -0.74 & 1.60 \\
\hline & $\mathrm{b}$ & 18.51 & 3.33 & 27.86 & 7.01 & 19.33 & 4.81 & 17.89 & 4.04 \\
\hline \multirow{3}{*}{4} & $\mathrm{~L}$ & 92.76 & 2.66 & 87.75 & 2.75 & 91.25 & 12.90 & 95.06 & 2.35 \\
\hline & $\mathrm{a}$ & -0.58 & 0.90 & 0.30 & 0.97 & -2.07 & 1.09 & -2.22 & 1.14 \\
\hline & $\mathrm{b}$ & 19.04 & 2.87 & 27.07 & 5.19 & 19.91 & 4.96 & 18.90 & 5.41 \\
\hline
\end{tabular}

Table 2: Mean and standard deviation (SD) and the results of statistical analysis for comparison of color differences $(\Delta \mathrm{E})$ between different treatment stages among the study groups (Group 1 demineralized teeth subjected to in-office bleaching, Group 2 demineralized teeth exposed to home bleaching, Group 3 sound teeth subjected to in-office procedure, Group 4 sound teeth exposed to home bleaching)

\begin{tabular}{|c|c|c|c|c|c|c|c|c|c|c|c|c|}
\hline \multirow{2}{*}{ Groups } & \multicolumn{2}{|c|}{$\Delta \mathrm{E} T 1-\mathrm{T} 2$} & \multicolumn{2}{|c|}{$\Delta \mathrm{E} T 1-\mathrm{T} 3$} & \multicolumn{2}{|c|}{$\Delta \mathrm{E} T 1-\mathrm{T} 4$} & \multicolumn{2}{|c|}{$\Delta \mathrm{E} T 2-\mathrm{T} 3$} & \multicolumn{2}{|c|}{$\Delta \mathrm{E} \mathrm{T2-T4}$} & \multicolumn{2}{|c|}{$\Delta \mathrm{E}$ T3-T4 } \\
\hline & Mean & SD & Mean & SD & Mean & SD & Mean & SD & Mean & SD & Mean & SD \\
\hline 1 & 15.52 & 5.04 & 8.01 & 5.04 & 4.61 & 2.23 & 21.26 & 12.41 & 19.57 & 3.83 & 6.42 & 18.12 \\
\hline 2 & 10.65 & 12.60 & 4.96 & 12.60 & 8.65 & 16.64 & 12.09 & 12.03 & 15.31 & 18.97 & 7.14 & 16.83 \\
\hline 3 & 12.44 & 8.89 & 8.36 & 8.89 & 6.00 & 3.83 & 14.45 & 14.55 & 13.97 & 7.11 & 5.37 & 16.63 \\
\hline 4 & 10.76 & 6.31 & 7.31 & 6.31 & 6.03 & 3.47 & 12.69 & 10.75 & 12.66 & 5.53 & 5.32 & 12.41 \\
\hline P value & 0.23 & & 0.732 & & 0.449 & & 0.049 & & 0.017 & & 0.966 & \\
\hline
\end{tabular}

healthy teeth. This indicates that the demineralized enamel is possibly more susceptible to absorb external stains than sound enamel. However, the difference in $\Delta$ ET1-T2 was not statistically significant among the study groups.

Following the bleaching, all the test groups displayed color improvement. The third color assessment (T3) was performed on the $8^{\text {th }}$ day after starting home bleaching procedure and following 2 sessions of laserassisted in-office bleaching, whereas the final color measurement (T4) was accomplished one day after completion of the bleaching treatments. The color changes between T1-T3, T1-T4, and T3-T4 stages were not significantly different among the study groups. However, the color change between $\mathrm{T} 2$ and T3 stages as well as the color change between T2 and T4 time points were significantly greater in demineralized teeth submitted to laser-assisted in-office bleaching (group 1) as compared to the other study groups. It is believed that HP diffuses through the enamel and dentin, producing free radicals that react with pigment molecules. 26) White spot lesions have a subsurface porosity in the 
enamel below the well mineralized surface layer. ${ }^{27-29)}$ This porosity may induce increased diffusion of oxidizing agents and more whitening effect on demineralized than sound teeth. The greater concentration of HP as employed in power bleaching could help to achieve a quicker and greater whitening effect on demineralized teeth compared to that occurs with home bleaching process

In the present study, the efficacy of both laserassisted in-office bleaching and home bleaching was similar on stained sound enamel. In contrast, the laserassisted in-office procedure was more effective than home bleaching in whitening demineralized enamel. It is difficult to compare the results of this study with that of previous investigations as most studies compared the whitening efficacy of different methods on healthy enamel. The findings of this study corroborate the results of some previous authors ${ }^{16}$ ) who found that both professional bleaching procedures were effective in whitening healthy teeth. In a clinical study, Mondelli et al. ${ }^{30)}$ concluded that LED/Diode laser activation of the bleaching agent can produce effective results in less time, compared to home-bleaching or in-office bleaching without light activation. The outcomes of this study, however, contradict the results of Zekonis et al. 31) who showed that the home applied procedure produced significantly more whitening effect than inoffice process during all active-treatment periods and follow-up visits.

The goal of power bleaching is to whiten the teeth efficiently, while minimizing any adverse effects on tooth structures. It is assumed that irradiation from a diode laser light source heats the HP, thereby accelerating the decomposition of hydrogen peroxide and formation of hydroxyl and oxygen free radicals 19, 20) and in this way leads to reduced treatment time and possibly enhances treatment results. The results of this study indicated faster and greater whitening effect on stained demineralized teeth by using the laser-assisted in-office bleaching compared to that of the home bleaching technique. Many patients are interested in obtaining whiter teeth quickly, thus laser-assisted inoffice bleaching could be recommend for them.

There is some controversy regarding the benefits of power bleaching compared to that of in-office bleaching without light activation. Son et al. 16) found that the diode laser irradiation improved the whitening effect of HP. Dominguez et al. ${ }^{32)}$ believed that for effective tooth whitening, the light source is more important than the bleaching agent. They found that activation with a LED light source should be considered the best option as a great color change was achieved with negligible increase in the pulp temperature. Luk et al. ${ }^{33)}$ and Suleiman et al. ${ }^{34)}$ proved that the application of different light sources and laser systems for activating the bleaching agent improved the success of overall tooth whitening. Gurgan et al. 35) indicated that in-office bleaching systems with or without light activation improved tooth color but bleaching with diode laser had the additional advantage of reducing tooth and gingival sensitivity. In contrast, Calatayud et al. ${ }^{36)}$ concluded that the use of diode laser negligibly improved the whitening efficacy of a 35\% HP gel. Strobl et al. ${ }^{37)}$ and Marcondes et al. ${ }^{38)}$ indicated that the additional activation of the HP-containing agent with a Nd:YAG laser was not associated with an enhanced bleaching performance. In a clinical study with a split-mouth design, Hein et al. ${ }^{39)}$ displayed that the three bleaching lights tested had no additional effect over the bleaching gel alone.

Clinically, lasers should be used with great care to prevent excessive increase in the pulp temperature. In this study, $2 \mathrm{~W}$ power was selected according to the recommendations of the manufacturer and the results of previous authors. ${ }^{14)}$ A 2 minute interval between laser exposures was designed to prevent from thermal damage to the pulp tissue. Another option is to use the laser in pulsed instead of continuous wave mode in order to allow tooth structures to cool down between irradiations and thus prevent from heat accumulation in the underlying tissues. ${ }^{40)}$ Other factors may also influence the temperature of the dental surface during laser-assisted bleaching such as the thickness of the bleaching gel, the duration of laser application, the distance between laser tip and tooth surface and the color of the bleaching gel. ${ }^{41)}$ A limitation of this study was that the effect of conventional in-office bleaching was not compared with that of laser-assisted in-office bleaching in order to test any benefit of laser treatment in the whitening process. Future studies should focus on evaluating the temperature of the tooth and pulp tissue using different laser powers and modes of irradiation. Further clinical studies are also warranted to compare the efficacy and long-term stability of laserassisted in-office bleaching with that of home bleaching and power bleaching using other light sources for treatment of white spot lesions.

\section{Conclusion}

Under the conditions used in this study:

1- The laser-assisted in-office bleaching produced faster and greater whitening effect on stained white spot lesions compared to that of the home 
bleaching technique and thus it could be recommended for patients with discolored demineralized teeth.

\section{References}

1: Heravi F, Ahrari F, Mahdavi M, and Basafa S (2014): Comparative evaluation of the effect of Er:YAG laser and low level laser irradiation combined with CPP-ACPF cream on treatment of enamel caries. Journal of Clinical and Experimental Dentistry,6:e121-126.

2: Behnan SM, Arruda AO, González-Cabezas C, Sohn W et al (2010): In-vitro evaluation of various treatments to prevent demineralization next to orthodontic brackets. American Journal of Orthodontics and Dentofacial Orthopedics,138:712.e711-717; discussion 712-713.

3: Ellwood RP, O'Mullane D (1995): Enamel opacities and dental esthetics. Journal of Public Health Dentistry, 55:171-176.

4: Akin M, Basciftci FA (2012): Can white spot lesions be treated effectively? The Angle Orthodontist, 82:770-775.

5: Lee JH, Kim DG, Park CJ, and Cho LR(2013): Minimally invasive treatment for esthetic enhancement of white spot lesion in adjacent tooth. The Journal of Advanced Prosthodontics,5:359-363.

6: Nahsan FP, da Silva LM, Baseggio W, Franco EB et al (2011): Conservative approach for a clinical resolution of enamel white spot lesions. Quintessence International,42:423-426.

7: Bussadori SK, do Rego MA, da Silva PE, Pinto MM et al (2004): Esthetic alternative for fluorosis blemishes with the usage of a dual bleaching system based on hydrogen peroxide at 35\%. The Journal of Clinical Pediatric Dentistry, 28:143-146

8: Knösel M, Attin R, Becker K, and Attin T (2007): External bleaching effect on the color and luminosity of inactive white-spot lesions after fixed orthodontic appliances. The Angle Orthodontist,77:646652.

9: Luna AJ, Nascimento CAO, Chiavone-Filho O (2006): Photodecomposition of hydrogen peroxide in highly saline aqueous medium. Brazilian Journal of Chemical Engineering, 23:341-349.

10: Berga-Caballero A, Forner-Navarro L, AmengualLorenzo J (2006): At-home vital bleaching: A comparison of hydrogen peroxide and carbamide peroxide treatments. Medicina Oral, Patologia Oral y Cirugia Bucal,11:E94-99.
2- The laser-assisted in-office bleaching and home bleaching displayed similar efficacy in stained sound enamel.

11: Haywood VB (1992): History, safety, and effectiveness of current bleaching techniques and applications of the nightguard vital bleaching technique. Quintessence International,23:471-488.

12: Joiner A (2006): The bleaching of teeth: A review of the literature. Journal of Dentistry,34:412-419.

13: Wetter NU, Barroso MC, Pelino JE (2004): Dental bleaching efficacy with diode laser and led irradiation: An in vitro study. Lasers in Surgury and Medicine,35:254-258.

14: Ghanbarzadeh M, Ahrari F, Akbari M, and Hamzei H (2015): Microhardness of demineralized enamel following home bleaching and laser-assisted in office bleaching. Journal of Clinical and Experimental Dentistry,7:e405-409.

15: Berger SB, Cavalli V, Ambrosano GM, and Giannini M (2010): Changes in surface morphology and mineralization level of human enamel following inoffice bleaching with 35\% hydrogen peroxide and light irradiation. General Dentistry, 58:e74-79.

16: Son JH, An JH, Kim BK, and Hwang IN et al (2012): Effect of laser irradiation on crystalline structure of enamel surface during whitening treatment with hydrogen peroxide. Journal of Dentistry, 40:941-948.

17: Shahabi M, Moosavi H, Gholami A, and Ahrari F (2012): In vitro effects of several surface preparation methods on shear bond strength of orthodontic brackets to caries-like lesions of enamel. European Journal of Paediatric Dentistry,13:197-202.

18: Sulieman M, Addy M, Rees JS (2003): Development and evaluation of a method in vitro to study the effectiveness of tooth bleaching. Journal of Dentistry,31:415-422.

19: Poosti M, Ahrari F, Moosavi H, and Najjaran $\mathrm{H}$ (2014): The effect of fractional CO2 laser irradiation on remineralization of enamel white spot lesions. Lasers in Medical Science,29:1349-1355.

20: Sulieman M, MacDonald E, Rees JS, and Addy M (2005): Comparison of three in-office bleaching systems based on 35\% hydrogen peroxide with different light activators. American Journal of Dentistry, 18:194-197.

21: Lia Mondelli RF, Garrido Gabriel TR, Piola Rizzante FA, Magalhães AC et al (2015): Do different 
bleaching protocols affect the enamel microhardness? European Journal of Dentistry,9:25-30.

22: Sari T, Celik G, Usumez A (2015): Temperature rise in pulp and gel during laser-activated bleaching: In vitro. Lasers in Medical Science,30:577-582.

23: Pretty IA, Aminian A, Davies RM, Davies RM et al (2006): Vital tooth bleaching in dental practice: 3 . Biological, dental and legal issues. Dental Update, 33: 422-4, 427-8, 431-2

24: Teruel Jde D, Alcolea A, Hernández A, Ruiz AJ (2015): Comparison of chemical composition of enamel and dentine in human, bovine, porcine and ovine teeth. Archives of Oral Biology,60:768775 .

25: Camargo MA, Marques MM, de Cara AA (2008): Morphological analysis of human and bovine dentine by scanning electron microscope investigation. Archives of Oral Biology,53:105-108.

26: Rotstein $\mathrm{L}$, and $\mathrm{Li} \mathrm{Y}$ : Tooth discolotation and bleaching. In: Ingle JI (ed) Endodontics. 2008, Hamilton, Ontario, pp 1383-1399.

27: Fejerskov O, Yaeger JA, Thylstrup A (1979): Microradiography of the effect of acute and chronic administration of fluoride on human and rat dentine and enamel. Archives of Oral Biology,24:123130.

28: Newbrun E (1960): Studies on the physical properties of fluorosed enamel. Ii. Microhardness. Archives of Oral Biology,12:21-27.

29: Shahabi M, Ahrari F, Mohamadipour H, Moosavi H (2014): Microleakage and shear bond strength of orthodontc brackets bonded to hypomineralized enamel following different surface preparations. Journal of Clinical and Experimental Dentistry 6:e110-5.

30: Mondelli RF, Azevedo JF, Francisconi AC, Almeida CM et al (2012): Comparative clinical study of the effectiveness of different dental bleaching methods - two year follow-up. Journal of Applied Oral Science, $20: 435-443$.

31: Zekonis R, Matis BA, Cochran MA, Al Shetri SE et al (2003): Clinical evaluation of in-office and athome bleaching treatments. Operative Dentistry, 28:114-121.
32: Domínguez A, García JA, Costela A, and Gómez C(2011): Influence of the light source and bleaching gel on the efficacy of the tooth whitening process. Photomedicine and Laser Surgury,29:5359.

33: Luk K, Tam L, Hubert M (2004): Effect of light energy on peroxide tooth bleaching. Journal of the American Dental Association,135:194-201; quiz 228-199.

34: Sulieman MA (2008): An overview of tooth-bleaching techniques: Chemistry, safety and efficacy. Periodontology 2000,48:148-169.

35: Gurgan S, Cakir FY, Yazici E (2010): Different light-activated in-office bleaching systems: A clinical evaluation. Lasers in Medical Science,25:817822.

36: Calatayud JO, Calatayud CO, Zaccagnini AO, and Box MJ (2010): Clinical efficacy of a bleaching system based on hydrogen peroxide with or without light activation. The European Journal of Esthetic Dentistry, 5:216-224.

37: Strobl A, Gutknecht N, Franzen R, Hilgers RD et al (2010): Laser-assisted in-office bleaching using a neodymium:Yttrium-aluminum-garnet laser: An in vivo study. Lasers in Medical Science, 25:503-509.

38: Marcondes M, Paranhos MP, Spohr AM, Mota EG et al (2009): The influence of the Nd:YAG laser bleaching on physical and mechanical properties of the dental enamel. Journal of Biomedical Materials Research. Part B, Applied Biomaterials, 90:388-395.

39: Hein DK, Ploeger BJ, Hartup JK,Wagstaff RS et al (2003): In-office vital tooth bleaching--what do lights add? Compendium of Continuing Education in Dentistry, 24:340-352.

40: Ahrari F, Heravi F, Fekrazad R, Farzanegan F et al (2012): Does ultra-pulse CO2 laser reduce the risk of enamel damage during debonding of ceramic brackets? Lasers in Medical Science,27:567-574.

41: Fornaini C, Lagori G, Merigo E, Meleti M et al (2013): Analysis of shade, temperature and hydrogen peroxide concentration during dental bleaching: In vitro study with the ktp and diode lasers. Lasers in Medical Science,28:1-6.

\section{[Acknowledgements]}

This article is based on an undergraduate thesis (No. 2586). The authors would like to thank the vice chancellor for research of Mashhad University of Medical Sciences for technical and financial support of this project.

\section{[Conflict of interest]}

The authors deny any conflict of interest. 